\title{
Polariton Motional Narrowing in Semiconductor Multiple Quantum Wells
}

\author{
J. J. Baumberg, ${ }^{1}$ A. P. Heberle, ${ }^{1}$ A. V. Kavokin, ${ }^{2}$ M. R. Vladimirova, ${ }^{2}$ and K. Köhler ${ }^{3}$ \\ ${ }^{1}$ Hitachi Cambridge Laboratory, Cavendish Laboratory, Madingley Road, Cambridge CB3 OHE, United Kingdom \\ ${ }^{2}$ Istituto Nazionale per la Fisica della Materia, Il Universita di Roma “Tor Vergata," I-00133 Roma, Italy \\ ${ }^{3}$ Fraunhofer-Institut für Angewandte Festkörperphysik, 79108 Freiburg, Germany
}

(Received 14 October 1997)

\begin{abstract}
We demonstrate a new fast-scan ultrafast coherent reflectivity technique that tracks the amplitude and phase of excitons in GaAs heterostructures at densities down to $4 \times 10^{6} \mathrm{~cm}^{-2}$. Observed growth in the exciton coherence over the first $1.5 \mathrm{ps}$ is direct evidence for the presence of interfering polariton modes. Polariton theory provides a good account of the data as the number of quantum wells is increased. Polariton motional narrowing of spectral lines from multiple quantum wells is demonstrated both experimentally and theoretically, annealing out the inhomogeneous broadening of the exciton energies. [S0031-9007(98)05867-0]
\end{abstract}

PACS numbers: 71.36.+c, 42.50.Md, 78.47.+p, 78.66.-w

The coherent properties of the light-semiconductor interaction are of strong interest because they influence the ultimate fate of light entering a semiconductor. This is especially true for illumination of the tightly bound electronhole pairs that form excitons below the direct band gap. The phase-coherent memory of electronic carriers produces an optical response strongly dependent on the previous state of the semiconductor, even at low carrier density. Coherent effects have been implicated in many interaction phenomena such as four-wave mixing [1,2], propagation effects [3,4], coherent control [5,6], Rayleigh scattering [7,8], and super-radiant photoluminescence [9]. Intentional separation of optically active layers enhances coherent couplings in microcavity or Bragg structures. However, in standard multiple quantum well (QW) structures, coupled modes of exciton and photon (polaritons) have not been considered particularly important. Exciton polaritons extend across the entire sample, while uncoupled excitons are localized within single QWs. In coherent optical experiments, only a few of the polariton modes (termed "bright") dominate the optical response [10]. Polaritons control how light encounters the semiconductor [11], and recently have been shown to suppress the influence of disorder in microcavities $[12,13]$.

In this paper we show directly that excitonic polaritons dominate the low temperature optical properties of most GaAs multiple QW samples of conventional design. Our results are based on a new ultrafast interferrometric technique for measuring the intensity of coherent reflections to 1 part in $10^{10}$ of the incident pulse at carrier densities, $n \sim 10^{8} \mathrm{~cm}^{-2}$. The reflectivity from a sample with 5 QWs shows a marked rise for the first $1.5 \mathrm{ps}$ which is unambiguously associated with polaritons not found in an equivalent sample with only a single QW. As we show in this Letter, the interference of different bright polariton modes can induce oscillations and even an initial rise in time-resolved reflection. In the presence of disorder, a quantum motional narrowing effect [14] appears due to exciton polaritons. Because they are delocalized over all the QWs, they average the potential disorder acting on excitons in different wells. This leads to the effective narrowing of the inhomogeneous exciton lines and a corresponding increase of the exciton polarization lifetime, with an increasing number of QWs. Here we demonstrate polariton motional narrowing in multiple QWs for the first time experimentally and theoretically.

The ultrafast coherent reflectivity technique derives from previous coherent control experiments [5] which measured the total carrier density photoexcited by two successive $100 \mathrm{fs}$ infrared pulses of actively controlled relative phase. Now we record the total reflectivity of the two pulses off the sample, $R=R_{0}+\Delta R(\tau)$, as a function of their delay $\tau$. The reflectivity of the second pulse is modified by the phase-coherent excitons injected by the first pulse. If the second pulse arrives in phase with the coherent excitons in the sample, then stimulated absorption occurs and $R$ is reduced [5,15]. Conversely, if the second pulse arrives half an exciton time period later $(\sim 1.3 \mathrm{fs})$, then stimulated emission occurs and the excitons are reextracted, thus increasing the reflectivity. Hence (for pulses short compared to the coherence decay time), $\Delta R$ oscillates in time delay with a period and decay which tracks the total electronic polarization, $\Delta R \propto P^{(\text {tot })}$, excited in the sample.

Figure 1(a) shows the Michelson interferometer used to create the pump pulse pair [15]. The delay is scanned repeatedly at a frequency of $4.2 \mathrm{~Hz}$, and the reflectivity versus delay is averaged over many scans. The interference of a He-Ne beam collinear with the infrared pulses allows tracking of the delay within 50 as, thus ensuring that the reflectivity fringes are not washed out by drift. Balanced subtraction of the reflected light from that incident on the sample suppresses laser noise and reduces the coherent spike at $\tau=0$ from direct temporal overlap of the two pulses. This time-domain Fourier transform technique is superior to alternative spectral interferometry $[16,17]$ because it directly tracks crucial weak signals at large $\tau$ which convolute into spectra as weak perturbations. 

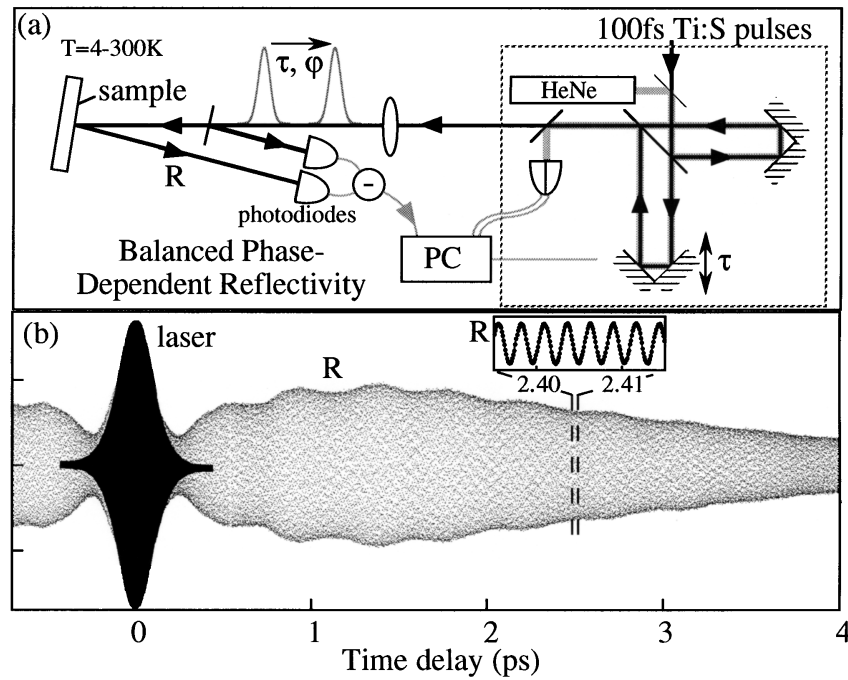

FIG. 1. (a) Fast-scan coherent reflectivity: Laser pulses are divided in the interferometer, recombined collinearly, and focused to a $200 \mu \mathrm{m}$ spot on the sample. The time delay between the pulses is rapidly scanned while tracked to an accuracy of $<50$ as. (b) Phase dependent reflectivity oscillations on the 5QW sample at $T=4 \mathrm{~K}$. The solid trace shows the direct interference of the laser pulses.

It is also preferred over conventional time-resolved upconversion, which is phase insensitive and has a lower signal to noise ratio at low density.

The samples used are high-quality single (SQW) and quintuple (5QW) $12 \mathrm{~nm}$ GaAs QWs separated by $12 \mathrm{~nm}$ $\mathrm{Al}_{0.3} \mathrm{Ga}_{0.7} \mathrm{As}$ barriers, although similar features have been seen in other structures. Figure 1(b) shows the reflectivity oscillations from a laser pulse detuned $4 \mathrm{meV}$ below the heavy-hole exciton in the 5QW sample at $T=4 \mathrm{~K}$, and $n \simeq 10^{8} \mathrm{~cm}^{-2}$. Both a $70 \%$ rise in the fringe amplitude and subsequent decay are weakly modulated by beats between light-hole and heavy-hole excitons. The general form of the signal varies little with detuning, apart from the strength of the beats. The $1.5 \mathrm{ps}$ signal rise is extremely robust and insensitive to the light polarization and pulse width. In a standard model (which includes propagation effects) it is completely unexpected since it would imply that the exciton coherence increases with time initially. As will be shown later, the rise is produced by the simultaneous excitation of two nondegenerate polariton modes with different initial phases which start to rephase as time progresses.

The coherent reflectivity rise is strongly dependent on the carrier density. Figure 2(a) shows the amplitude of the fringes in $R(\tau)$ at a range of densities. Below $n \simeq$ $10^{8} \mathrm{~cm}^{-2}$ the fringe amplitude follows the same shape, but higher densities wash out the early rise. The phase dynamics of the interference oscillations are even more density dependent and will be discussed elsewhere. This density dependence explains why such effects have not been previously noted-nonlinear measurements rarely

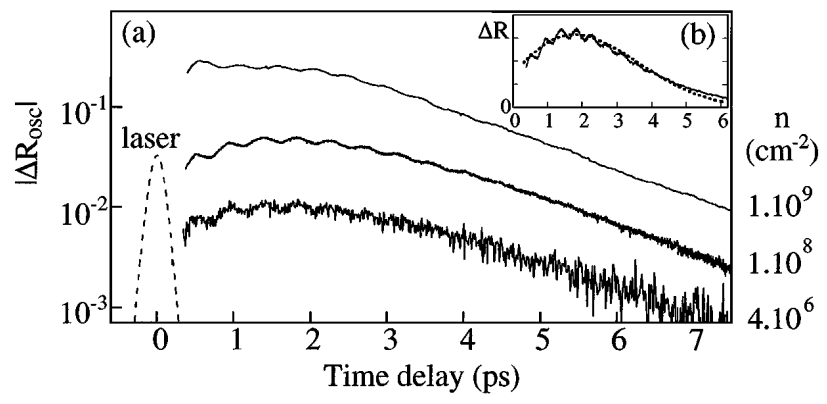

FIG. 2. (a) Coherent reflection amplitude (vertically offset) from the 5QW sample vs carrier density, $T=4 \mathrm{~K}$. (b) Rise and decay fit by a simple polariton interference model (dashed) described in the text.

escape the higher density regime. In contrast, the signal here is linear in the laser intensity, and allows exciton phase spectroscopy at much lower powers. It corresponds to four-wave mixing with zero intersection angle of the two pulses, but tracks the total sample polarization because it does not average over the phase information. Four-wave mixing at intermediate densities on the same sample matches the coherent reflectivity, implying that the third order polarization, $P^{(3)}$, simply tracks the total polarization.

To focus on possible polariton origins for the signal, we examine a range of samples of different design. Figure 3 shows the signals from the SQW, 5QW, a metal mirror (to estimate the laser pulse pedestal) and a 25QW sample with $18 \mathrm{~nm}$ GaAs QWs between $10 \mathrm{~nm} \mathrm{Al}_{0.35} \mathrm{Ga}_{0.65} \mathrm{As}$ barriers [18]. The latter sample is used to provide direct comparison with resonant Rayleigh scattering data recently taken on it [8]. The SQW sample shows no rise before the reflected amplitude decays. In the theory described below, signals from both the SQW and 5QWs can be fit with the same material parameters.

The effect of polaritons can be described in a simplified picture as follows: The optical coupling of a number of QWs leads to an equal number of polariton modes. These are split in energy from each other, and experience different refractive indices. Light reflected by the different polariton modes will therefore have different optical path delays. Because of the different polariton energies there will be a beating of the modes, which can cause a rise in reflection intensity since the modes are not in phase initially. Homogeneous and inhomogeneous broadening will wash out the beating at later times leaving only the initial rise.

More rigorously, our approach is based on solving Maxwell equations for light incident on the structure in the vicinity of the exciton resonance frequency. Because of inevitable fluctuations in the confinement potential ("lateral" disorder), the exciton energies are distributed [19], and approximated by an asymmetric Gaussian with broadening parameters $\Delta_{1,2}$ for the low- and high-energy wings of the distribution. The wave vector of light is 


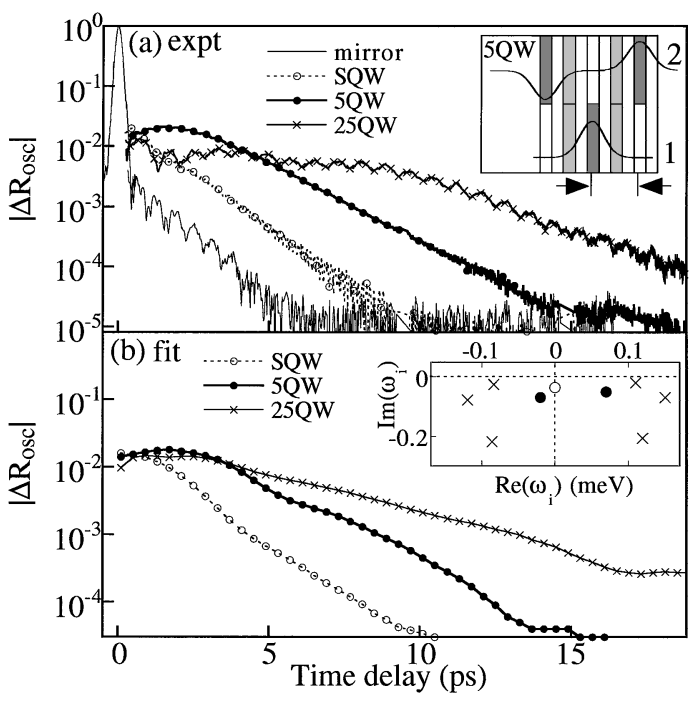

FIG. 3. (a) Experimental reflectivity amplitude from a metal mirror, 1, 5, and $25 \mathrm{QW}$ samples. $n \simeq 10^{8} \mathrm{~cm}^{-2}, T=$ $4 \mathrm{~K}$. Inset: Schematic polariton envelopes showing two bright modes which couple excitons vertically between 5 different QWs. (b) Fits to the same samples using radiative coupling rate $=26 \mu \mathrm{eV}$, homogeneous linewidth $=0.1 \mathrm{meV}$. Inhomogeneous linewidths for the SQW and 5QW of $0.4 \mathrm{meV}$ below and $0.7 \mathrm{meV}$ above the exciton center were used, while $0.1 \mathrm{meV}$ was used for both in the $25 \mathrm{QW}$ sample. Inset: Dominant bright poles of $r(\omega)$ relative to $\omega_{\mathrm{ex}}=1543 \mathrm{meV}$.

conserved in the plane of the QW, and excitons with different resonance frequencies contribute to the dielectric polarization of the QWs, $\tilde{\chi}$. This model, while approximate, describes with good accuracy both $\mathrm{cw}$ and time-resolved reflection experiments [20]. Its advantage is that it yields analytical expressions for measurable optical characteristics. In particular, the amplitude reflection coefficient for a single QW has the form $r(\omega)=\frac{i \alpha \tilde{\chi}}{1-i \alpha \tilde{\chi}}$, where $\alpha$ is a measure of the exciton-photon coupling strength. A transfer matrix formalism is then used to derive the reflection coefficient from a number of stacked QWs. The Fourier transform of this quantity predicts the measured time-resolved reflection signal.

This semiclassical approach does not involve a priori the concept of quantum particles (excitons, photons, and polaritons). However, it yields the full range of polariton effects, including the quantum motional narrowing effect described below. Indeed, the calculated time-resolved reflection spectra for the samples under study shown in Fig. 3(b) display excellent agreement with the experimental data.

We emphasize that the signal decay time clearly increases with an increasing number of QWs. This remarkable tendency obtained both theoretically and experimentally is in apparent contradiction with existing super-radiance concepts for multiple QWs which ignored disorder effects [10]. According to this theory, in a structure containing $x$ QWs there exist $x$ polariton modes with slightly different frequencies and strongly different oscillator strengths. A few of these modes have an oscillator strength larger than an exciton in a single QW and are termed bright or "super-radiant," while others are "dark" or "quasidark" having an oscillator strength less than a single $\mathrm{QW}$ exciton. The complex eigenfrequencies $\omega_{i}$ of the two bright modes in the 5QW sample, the six bright modes in the $25 \mathrm{QW}$ sample, and the lone mode in the single QW (calculated with no disorder) are shown in the inset of Fig. 3(b). They have been obtained as poles of the reflection coefficient of the relevant structures. The size of $\operatorname{Im}\left(\omega_{i}\right)$ is proportional to the oscillator strength and decay rate of the corresponding polariton mode. The decay time of the time-resolved reflection is governed by super-radiant modes: it decreases with an increase of the oscillator strength in these modes, thus it should decrease with an increasing number of QWs (contrary to what is observed).

Our explanation of this discrepancy is as follows. There are two radiation regimes in multiple QWs: a superradiance regime and a motional narrowing regime. The former is realized if the radiative decay rate of superradiant modes exceeds the inhomogeneous broadening of excitons. In this case, the decay of the reflected signal is governed by bright polaritons, and the super-radiance theory is valid. In the motional narrowing regime, the inhomogeneous broadening exceeds the decay rate of bright polaritons and governs the coherence decay rate. The stronger the disorder, the shorter the coherence memory time. Polaritons, i.e., extended exciton-photon modes spread over the entire structure, still exist, but their role in this regime is in averaging the disorder. This "vertical" averaging (along the growth axis) becomes more efficient with an increasing number of QWs. Consequently, the decay time increases with increasing the number of QWs. Motional narrowing clearly improves the perceived growth quality of multiple QWs yielding narrower spectral lines and longer polarization decays.

Strong proof of the dominant role of polaritons in our experiments is the initial rise of the time-resolved signal for the 5QW sample [Fig. 2(a)]. It is easily understood in terms of the two interfering bright polariton modes existing in the structure [Fig. 3(b), inset, -]. Since the phase of the amplitude reflection coefficient changes from $-\pi / 2$ to $\pi / 2$ across the exciton resonance, these nondegenerate modes at $\omega_{1,2}$ emit light with different phases [where $\delta \phi=\phi\left(\omega_{1}\right)-\phi\left(\omega_{2}\right)$ ]. Intuitively the different phases of the two modes can be understood from the different effective optical depths produced by their envelopes [depicted in Fig. 3(a), inset]. In this case the amplitude of emitted light decays as $\quad P^{\text {(tot) }} \propto\left\{\cos \left(\omega_{1} t\right)+\cos \left(\omega_{2} t+\delta \phi\right)\right\} \exp (-t / \tau)$. Clearly, it initially increases if $\delta \phi>\pi / 2$. Figure 2(b) shows the time-resolved reflection calculated within this simplified model, which agrees qualitatively with the data. The fit of the spectra for $1,5 \mathrm{QWs}$ within the accurate model [Fig. 3(b)] gives the best 


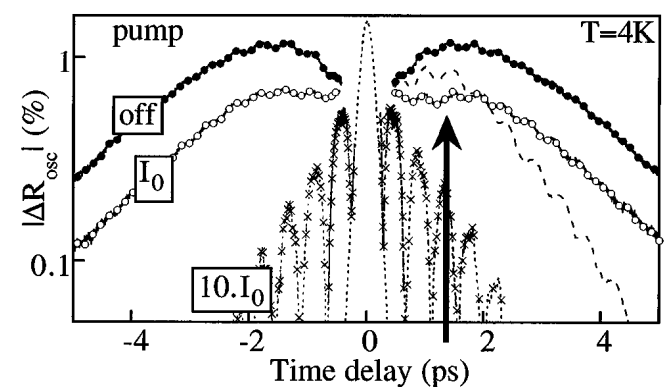

FIG. 4. Reflection amplitude in the absence and presence of an extra pump pulse arriving after $1.2 \mathrm{ps}$ (arrow), $I_{0} \simeq$ $10^{8} \mathrm{~cm}^{-2}$. With the pump off, the signal is symmetrical around zero delay because the phase-tracked pulses are identical. The pump should break this symmetry if there is a transient effect. Dashed curve is at $T=20 \mathrm{~K}$ with no pump.

agreement for the expected asymmetric exciton resonance line shape $\left(\Delta_{1}=0.4 \mathrm{meV}, \Delta_{2}=0.7 \mathrm{meV}\right)$. Such spectral asymmetry also arises from microscopic models of the confinement potential $[13,19]$ and is a consequence of the finite exciton mass in the plane of the QW.

Light interacts with semiconductors only through the polariton modes, so the lowest order optical nonlinearities will depend on polariton interactions. Raising the carrier density or temperature washes out polaritons at carrier densities well below the threshold for exciton-exciton interactions $\left(>10^{9} \mathrm{~cm}^{-2}\right)$. To confirm this, the experiment is modified by adding a third pump pulse focused to the same spot on the sample and timed to arrive $1.2 \mathrm{ps}$ after the first phase-coherent pulse. Figure 4 shows the reflection amplitude at three pump densities. The rise is destroyed and the polaritons eliminated at higher powers. This implies that excitons now phase scatter before they can reabsorb light emitted into each polariton (i.e., homogeneous broadening exceeds the polariton splittings). Moreover, polariton effects are suppressed both before and after the arrival of the pump pulse. Thus, the lowest order nonlinearity cannot be the exciton-exciton interaction which would only turn on when the pump pulse arrives. Instead, we suggest that acoustic phonons are responsible. Relaxing carriers emit acoustic phonons which persist over time scales longer than the time between pulses from the mode-locked laser. Scattering by acoustic phonons increases the homogeneous linewidth of the excitons, reducing the polariton contributions. Similar effects are seen by raising the sample temperature to $T=20 \mathrm{~K}$; however, it is still to be determined whether the acoustic phonons are in thermal equilibrium and how the "dynamic" disorder they produce disturbs the polariton coupling.

Optical spectroscopies on weakly excited semiconductor heterostructures at low temperature should be consid- ered from a polariton perspective. As a demonstration of these ramifications the $25 \mathrm{QW}$ sample shows strongly nonexponential decay in both experiment and theory [Figs. 3(a) and 3(b)] from six interfering bright polaritons. Resonant Rayleigh scattering from this sample at low densities also showed a corresponding nonexponential rise [8]. Thus, more appropriate models of the resonant Rayleigh scattering must incorporate polaritonic interference and motional narrowing effects.

In summary, exciton polariton effects dominate timeresolved coherent reflectivity measurements on standard multiple QW samples at low temperature and carrier density. Beating is observed between two bright polariton modes of initially different phase. Both experiment and theory clearly demonstrate that polaritons can partially average out the inhomogeneous spectrum of excitons, with implications for future structure designs. Polaritons also affect most other features of optical spectroscopy on quantum wells, which should be reevaluated in this light.

We happily acknowledge fruitful discussions with C. Andreani, B. Devaud, S. Haacke, and R. Phillips.

[1] M. Hübner et al., Phys. Rev. Lett. 76, 4199 (1996).

[2] S. Weiss, M.-A. Mycek, J.-Y. Bigot, S. Schmitt-Rink, and D. S. Chemla, Phys. Rev. Lett. 69, 2685 (1992).

[3] T. Stroucken et al., Phys. Rev. Lett. 74, 2391 (1995).

[4] D. Frölich et al., Phys. Rev. Lett. 67, 2343 (1991).

[5] A. P. Heberle, J. J. Baumberg, and K. Köhler, Phys. Rev. Lett. 75, 2598 (1995).

[6] D. S. Citrin, Phys. Rev. Lett. 77, 4596 (1996).

[7] H. Wang, J. Shah, T.C. Damen, and L. N. Pfeiffer, Phys. Rev. Lett. 74, 3065 (1995).

[8] S. Haacke, R. A. Taylor, R. Zimmermann, I. Bar-Joseph, and B. Deveaud, Phys. Rev. Lett. 78, 2228 (1997).

[9] D. Weber et al., J. Opt. Soc. Am. B 13, 1241 (1996).

[10] G. Björk et al., Phys. Rev. B 52, 17310 (1995); D. S. Citrin, Solid State Commun. 89, 139 (1994).

[11] D. S. Citrin, Phys. Rev. B 50, 5497 (1994).

[12] D. M. Whittaker et al., Phys. Rev. Lett. 77, 4792 (1996).

[13] V. Savona et al., Phys. Rev. Lett. 78, 4470 (1997).

[14] See, for example, A. Abragam, The Principles of Nuclear Magnetism (Clarendon, Oxford, 1961), p. 446.

[15] J. J. Baumberg, A.P. Heberle, K. Köhler, and A. V. Kavokin, Phys. Status Solidi (b) 204, 9 (1997).

[16] L. Lepetit, G. Chériaux, and M. Joffre, J. Opt. Soc. Am. B 12, 2467 (1995).

[17] W. Walecki, D. N. Fittinghoff, A. L. Smirl, and R. Trebino, Opt. Lett. 22, 81 (1997).

[18] Loaned in great spirit by the EPFL group.

[19] D. S. Citrin, Phys. Rev. B 47, 3832 (1993).

[20] L. C. Andreani, G. Panzarini, A. V. Kavokin, and M. R. Vladimirova, Phys. Rev. B 57, 4670 (1998). 